\title{
High-dose dobutamine stress SSFP cine MRI at 3 Tesla with patient adaptive local RF shimming using dual-source RF transmission
}

\author{
Alexander Berger ${ }^{1 *}$, Bernhard Schnackenburg ${ }^{2}$, Christopher Schneeweis ${ }^{1}$, Sebastian Kelle ${ }^{1}$, Christoph Klein ${ }^{1}$, \\ Marc Kouwenhoven ${ }^{3}$, Eckart Fleck', Rolf Gebker ${ }^{1}$
}

From 15th Annual SCMR Scientific Sessions

Orlando, FL, USA. 2-5 February 2012

\section{Background}

Image quality of cine imaging using steady state free precession (SSFP) sequences at $3 \mathrm{~T}$ is insufficient due to increased RF-inhomogeneity (B1 field) and the high sensitivity of SSFP sequences to off-resonance artefacts. Recently, the introduction of a dual source RF transmission system with patient-adaptive local RF-shimming has led to a significant improvement of image quality of SSFP imaging at $3 \mathrm{~T}$.

The objective of this study was to prospectively evaluate the feasibility, image quality and diagnostic accuracy of high-dose dobutamine stress magnetic resonance imaging (DSMR) at 3T comparing dual-source versus single-source transmit technology.

\section{Methods}

DSMR was performed in 44 patients with each participant undergoing cine imaging at rest and during dobutamine infusion using both dual- and single-source transmit technology.

B1-maps and measurements of contrast to noise ratio (CNR) were evaluated to quantify the effect of RF calibration in both transmission modes.

Analysis of image quality $(0=$ non diagnostic, $1=$ severe artifact, $2=$ slight artifact, $3=$ no artifact) and wall motion was performed at rest and at maximum stress comparing single- and dual-source technology.

CAD was defined on invasive coronary angiography as the presence of $\geq 70 \%$ stenosis.

'Department of Internal Medicine/Cardiology, German Heart Institute Berlin, Berlin, Germany

Full list of author information is available at the end of the article

\section{Results}

The mean percentage of the intended flip angle within the heart increased from $88 \% \pm 9.1$ with single-source to $103 \% \pm 5.6$ with dual-source $(\mathrm{p}<0.001)$. Deviation of the flip angle from the base to the apex along the pseudo-long axis decreased from $29.8 \% \pm 12.9 \%$ with single-source to $12.8 \% \pm 7.2 \%$ with dual-source.

CNR increased for dual-source vs. single-source especially pronounced at the apex $(63.4 \pm 24.2$ vs. $36.5 \pm$ $16.5, \mathrm{p}<0.001)$ but also at the base $(50.1 \pm 14.8$ vs. 39.3 $\pm 15.8, \mathrm{p}<0.001$ ).

Image quality of dual-source was higher than singlesource both at rest $(2.8 \pm 0.5$ vs. $2.6 \pm 0.7, \mathrm{p}<0.001)$ and stress $(2.5 \pm 0.7$ vs. $2.0 \pm 1.0, \mathrm{p}<0.001)$. The number of segments with either severe artifacts or non-diagnostic image quality at stress was $27 \%$ using single-source compared to only $8 \%$ using dual-source (figure 1 ).

No significant differences between dual-source DSMR and single-source DSMR were seen regarding sensitivity $(92 \%$ vs. $83 \%, \mathrm{p}=0.38)$ and specificity $(88 \%$ vs. $50 \%$, $\mathrm{p}=0.25)$ due to the relatively small patient cohort. Diagnostic accuracy of dual-source DSMR (90\%) was significantly higher than single-source DSMR (77\%) $(\mathrm{p}=0.006)$ (figure 2).

\section{Conclusions}

We demonstrated that using a dual-source transmit technology in a standard DSMR protocol is feasible in a 3T environment. Furthermore, the dual-source transmit technology provides better image quality and higher diagnostic accuracy compared to single-source transmit technology. 

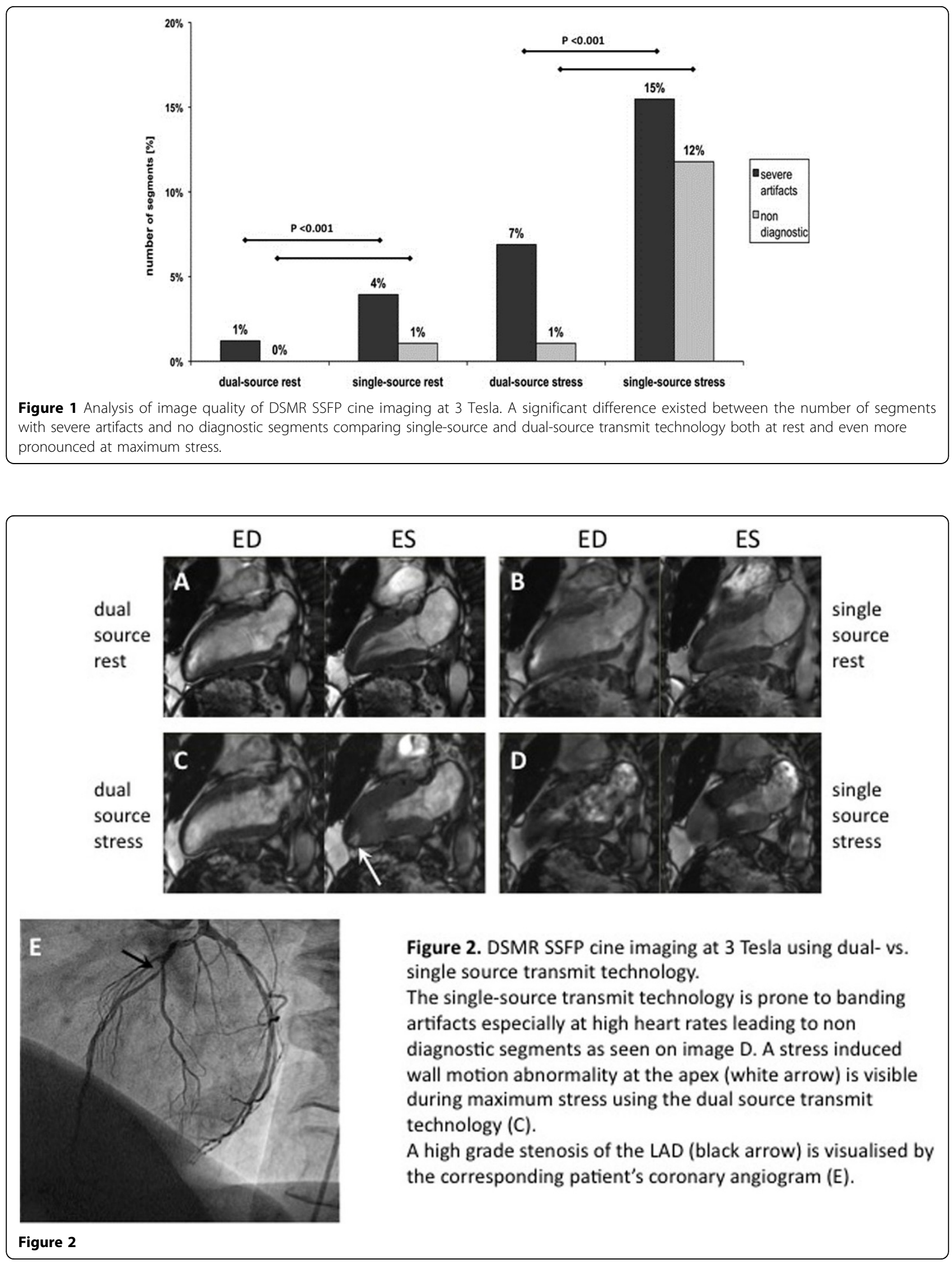


\section{Funding}

None.

\section{Author details}

${ }^{1}$ Department of Internal Medicine/Cardiology, German Heart Institute Berlin, Berlin, Germany. ${ }^{2}$ Philips Healthcare, Hamburg, Germany. ${ }^{3}$ Philips Healthcare, Best, Netherlands.

Published: 1 February 2012

doi:10.1186/1532-429X-14-S1-P252

Cite this article as: Berger et al:: High-dose dobutamine stress SSFP cine

MRI at 3 Tesla with patient adaptive local RF shimming using dual-

source RF transmission. Journal of Cardiovascular Magnetic Resonance

2012 14(Suppl 1):P252.

Submit your next manuscript to BioMed Central and take full advantage of:

- Convenient online submission

- Thorough peer review

- No space constraints or color figure charges

- Immediate publication on acceptance

- Inclusion in PubMed, CAS, Scopus and Google Scholar

- Research which is freely available for redistribution

Submit your manuscript at www.biomedcentral.com/submit
Ciomed Central 